\title{
Electron/Ion Channeling Contrast Imaging and Grayscale Image Analysis Using 3C-SiC Twin Structures
}

\author{
Tomoko Borsa ${ }^{1}$ and Bart Van Zeghbroeck ${ }^{2}$ \\ 1. Nanomaterials Characterization Facility, University of Colorado Boulder, Boulder, USA. \\ 2. Dept. of Electrical, Computer and Energy Engineering, University of Colorado Boulder, Boulder, \\ USA.
}

Channeling is the tendency of an incident beam of charged particles to penetrate deeper along the orientations of crystallographic planes with lower potential energies. The incident beam can be composed of electrons or ions, but these are expected to behave differently because of the different signs of charge and masses [1]. The channeling effect is known to be one of the main causes of image contrast in electron/ion microscopy. Typically, channeling contrast imaging (CCI) is performed using a scanning electron microscope equipped with a backscatter electron detector and/or forescattered electron detector, and they are used for the visualization of surface and subsurface defects in semiconductors and metals $[2,3]$. When the particles interact with the matter, secondary electrons (SEs) are also generated. However, those SEs created by the deeply penetrated channeling particles are not likely to reach the surface and be detected by a standard secondary electron detector (SED), therefore the area that matches the channeling condition appears darker. Alternatively, in the area that does not align well with the channeling orientation, SEs are generated near the surface resulting in the brighter contrast [4]. The variation of contrast could be used to determine crystal orientations [5].

In this study, 3C-SiC thin films with twin structures associated with double positioning boundaries, grown on (0001) 6H-SiC substrates, were used because this material was proven to be applicable for both electron/ion CCI-SED techniques [6]. The twin structures were found to be extremely useful to evaluate CCI since the difference in grayscale medians between the type I and II regions could be used as the parameter to quantify the channeling effect instead of the signal contrast itself, which was difficult [7]. The typical geometry of the experimental setup in Figure 1 and the stereographic projection of a 3C-SiC crystal on (111) in Figure 2 are shown. Briefly, the sample was mounted flat on a stub, and rotated so that one of the twin boundaries became parallel to the horizontal scanning direction. This rotation was set to be $\mathrm{R}=0^{\circ}$ in Figure 2. Then, the stage was tilted and SE images were recorded. When the channeling effect occurs, the type I and type II areas should have different contrast. The overall flow of image analysis is explained in Figure 3. After the images were collected, the tilt correction was required, and the 256-level grayscale distribution was obtained. If the separation in grayscale between the two types was large enough, two peaks would show up in the grayscale distribution. However, those often overlap, so small area analysis might be required as an additional step.

Figure 4 shows the result of the ECCI-SED analysis when the stage tilt was changed with the fixed rotation of $\mathrm{R}=0^{\circ}$ (tilting along with the red arrow in Figure 2) and $\mathrm{R}=180^{\circ}$ (the blue arrow in Figure 2). The grayscale median difference has the maximum $\left(\mathrm{R}=0^{\circ}\right)$ and minimum $\left(\mathrm{R}=180^{\circ}\right)$ at the tilt angle of $35^{\circ}$, which corresponds to the $<011>$ direction. ICCI-SED also demonstrated the maximum grayscale difference, more than 100 in the grayscale, with the tilt angle of $35^{\circ}$ from the incident ion beam. When the stage was rotated by $180^{\circ}$, the inversion of the contrast was observed. This was expected because these two regions in this twin structure are identical except for being rotated by $60^{\circ}$ and the the $<111>$ has a three-fold symmetry as shown in Figure 2. It is worth noting that ICCI could provide much larger grayscale 
difference than ECCI, and that screw dislocations were easily detectable with ICCI. However, the ion beam was more damaging on the samples, and this was one of the disadvantages.

Conclusion: The CCI techniques were applied on the 3C-SiC with the twin structure, and using this material found to be convenient in improving the grayscale image analysis.

References:

[1] J. C. H. Spence, in Electron Diffraction Techniques Volume 1", ed. J. M. Cowley, (Oxford University Press, New York) p.465.

[2] Y. N. Picard and M. E. Twigg, J. Appl. Phys. 104 (2008) p.124906.

[3] S. Zaefferer and N.-N. Elhami, Acta Materialia 75 (2014) p.20.

[4] Y. Yahiro et al, J. Electron Microsc. 53 (2004) p.571.

[5] R. E. Franklin et al, J. Mater. Sci. Lett. 7 (1988) p.39.

[6] T. Borsa et al, Microanal. 23 (Suppl 1) (2017) p.576.

[7] D. L. Barr and W. L. Brown, J. Vac. Sci. Technol. B 13 (1995) p.2580.

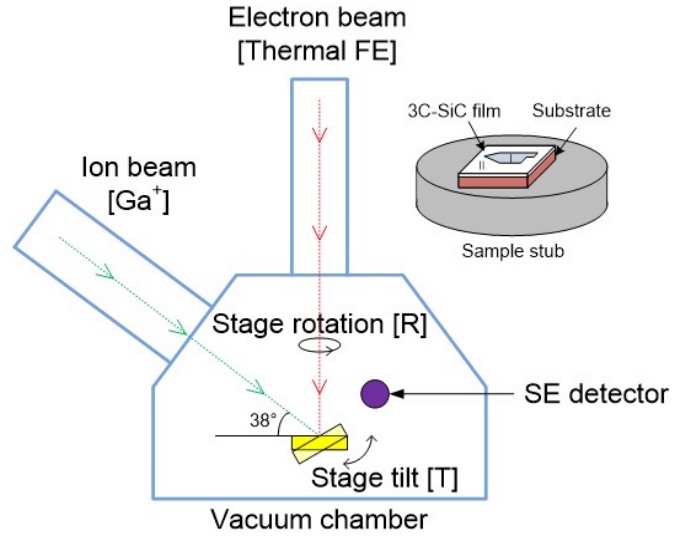

Figure 1. The experimental setup

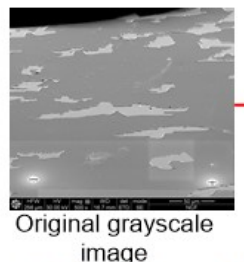

(a) Bimodal analysis

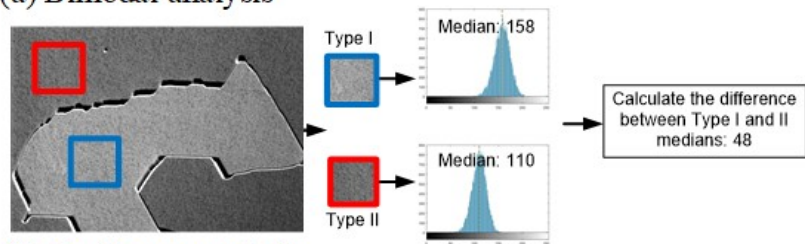

(b) Small area analysis

Figure 3. The flow of grayscale image analysis

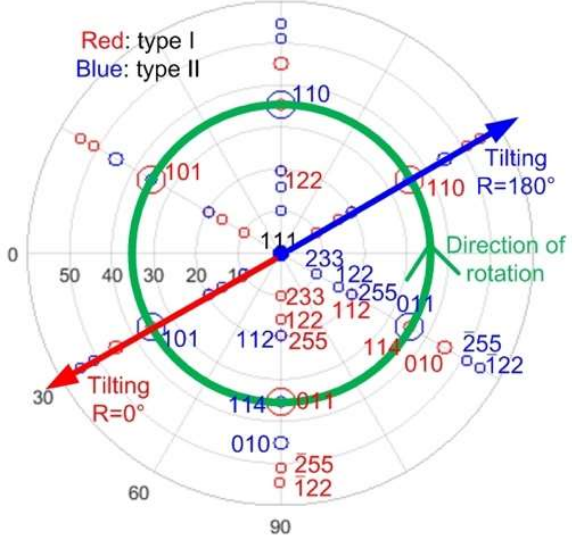

Figure 2. The stereographic projection of $3 \mathrm{C}-\mathrm{SiC}$ crystal

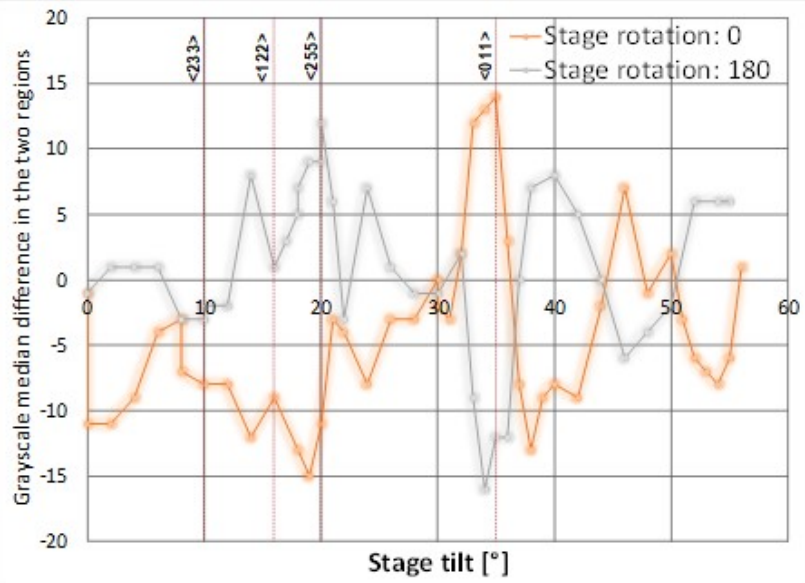

Figure 4. The ECCI-SED results. $(5 \mathrm{kV}, 98 \mathrm{pA})$ 